\title{
A comparison of impedance boundary conditions for flow acoustics
}

\section{Gwénaël Gabard}

Institute of Sound and Vibration Research, University of Southampton, UK

\section{A R T I C L E I N F O}

\section{Article history:}

Received 12 September 2012

Accepted 11 October 2012

Handling Editor: Y. Aurégan

Available online 10 November 2012

\begin{abstract}
A B S T R A C T
Acoustic liners remain a key technology for reducing community noise from aircraft engines. The choice of optimal impedance relies heavily on the modeling of sound absorption by liners under grazing flows. The Myers condition assumes an infinitely thin boundary layer, but several impedance conditions have recently been proposed to include a small but finite boundary layer thickness. This paper presents a comparison of these impedance conditions against an exact solution for a simple benchmark problem and for parameters representative of inlet and bypass ducts on turbofan engines. The boundary layer thickness can have a significant impact on sound absorption, although its actual influence depends strongly on the details of the incident sound field. The impedance condition proposed by Brambley seems to provide some improvements in predicting sound absorption compared to the Myers condition. The boundary layer profile is found to have little influence on sound absorption.
\end{abstract}

(c) 2012 Elsevier Ltd. All rights reserved.

\section{Introduction}

Acoustic liners are the most common technology used to reduce noise emissions from aircraft engines. Yet further improvements in their design will be required to support future evolutions of turbofans such as ultra-high bypass ratios and shorter nacelles. When predicting the efficiency of acoustic treatments for such applications, one has to model not only the interaction of the liner with the sound field but also the effects of the boundary layer of the grazing flow. This modifies the propagation of sound and can induce hydrodynamic oscillations and instabilities that interact with the liner and the sound field.

The impedance condition derived by Ingard [1] and generalized by Myers [2] has been the standard model to describe the effects of an infinitely thin boundary layer (BL) on sound absorption. But several limitations have become apparent. In parallel with experimental observations of instabilities developing over liners with grazing flows [3,4], the properties and stability of surface waves described by the Myers condition were also studied [5,6]. This led to the observation that the Myers condition is in fact ill-posed in the time domain due to the unbounded growth rate of the instability at high frequencies [7]. In addition, comparison with solutions with a finite boundary layer thickness has shown that this parameter can be significant [8-10]. Indeed, measurable discrepancies have been observed between experimental data and theoretical predictions (for instance in the context of impedance eduction methods [11]), suggesting that the accuracy of the Myers condition might not be sufficient for some practical applications.

In response to these findings, modified Myers conditions have recently been proposed to address the well-posedness issue. The model proposed by Rienstra and Darau [12,13] includes a small but finite boundary layer thickness $\delta$ and is derived by neglecting compressibility. Independently Brambley [14] derived a different impedance condition by using

E-mail address: gabard@soton.ac.uk 
matched asymptotic expansions based on the small parameter $\delta$. These two models are well posed in the time domain and provide improved descriptions of the hydrodynamic stability of the boundary layer (see also the recent discussion by Marx [15]). The introduction of the boundary layer thickness $\delta$ as an additional parameter offers the potential for more accurate predictions of the sound absorption. This is the topic of the present paper which aims to compare the Myers and modified Myers conditions against an exact solution to discuss the importance of the boundary layer thickness in practical applications and to assess how well the modified impedance conditions can capture these effects.

The next section describes the benchmark problem used for this comparison. Section 3 introduces some special cases to assess the consistency of the impedance conditions. Section 4 presents and discusses the results of the comparison.

\section{Plane wave reflection by a lined surface}

We consider a three-dimensional problem in the half-space $y>0$ with a uniform, subsonic mean flow, with Mach number $M$ in the $x$ direction, as illustrated in Fig. 1a. The sound field has an $\mathrm{e}^{+\mathrm{i} \omega t}$ time dependence. A boundary with uniform impedance $Z$ is located at $y=0$. All variables are non-dimensionalized using the sound speed $c_{\infty}$, the mean flow density $\rho_{\infty}$ and a length scale $L$. The velocity potential $\phi$ satisfies the convected Helmholtz equation

$$
\frac{\mathrm{d}_{0}^{2} \phi}{\mathrm{d} t^{2}}-\nabla^{2} \phi=0
$$

where $\mathrm{d}_{0} / \mathrm{d} t=\mathrm{i} \omega+M \partial / \partial x$ is the material derivative in the mean flow. We consider an incident plane wave reflected by the lined surface, so we assume a solution of the form

$$
\phi=A \exp \left(-\mathrm{i} k \boldsymbol{\theta}_{i} \cdot \mathbf{x}\right)+B \exp \left(-\mathrm{i} k \boldsymbol{\theta}_{r} \cdot \mathbf{x}\right),
$$

where the directions of the incident and reflected plane waves are given by

$$
\boldsymbol{\theta}_{i}=(\sin \vartheta \cos \varphi,-\cos \vartheta, \sin \vartheta \sin \varphi)^{T}, \quad \boldsymbol{\theta}_{r}=(\sin \vartheta \cos \varphi, \cos \vartheta, \sin \vartheta \sin \varphi)^{T},
$$

where the angles $\vartheta$ and $\varphi$ are defined in Fig. 1a. The acoustic wavenumber is $k=\omega / D$ where $D=1+M \sin \vartheta \cos \varphi$ is the Doppler factor associated with the effect of the mean flow on the acoustic wavelength. From the velocity potential $\phi$ we can obtain the acoustic pressure $p=-\mathrm{d}_{0} \phi / \mathrm{d} t$ and the velocity components $u=\partial \phi / \partial x, v=\partial \phi / \partial y$ and $w=\partial \phi / \partial z$.

With an appropriate boundary condition at $y=0$ we can calculate the reflection coefficient $R=B / A$. It is also convenient to consider the absorption coefficient $\alpha$ which is defined as the ratio between the acoustic power absorbed by the liner and the incident acoustic power. They are given by

$$
I_{\mathrm{abs}}=\frac{\omega^{2} \cos \vartheta}{2 D}\left(|A|^{2}-|B|^{2}\right), \quad I_{\mathrm{inc}}=\frac{\omega^{2} \cos \vartheta}{2 D}|A|^{2} .
$$

So the absorption coefficient is simply $\alpha=1-|R|^{2}$.

For all the boundary conditions considered here the reflection coefficient will be of the form

$$
R=\frac{Z\left(D \cos \vartheta+\mathrm{i} T_{1}\right)-1+\mathrm{i} T_{0}}{Z\left(D \cos \vartheta-\mathrm{i} T_{1}\right)+1+\mathrm{i} T_{0}},
$$

where $T_{0}$ and $T_{1}$ are real-valued parameters.

We will also consider the two-dimensional case when the waves propagate along the $x$-axis (that is $\varphi=0$ or $\pi$ ). In this case we introduce the angle of incidence $\theta$, as shown in Fig. 1b, and we have

$$
R=\frac{Z\left(D \sin \theta+\mathrm{i} T_{1}\right)-1+\mathrm{i} T_{0}}{Z\left(D \sin \theta-\mathrm{i} T_{1}\right)+1+\mathrm{i} T_{0}}
$$

with $D=1+M \cos \theta$.

(a)

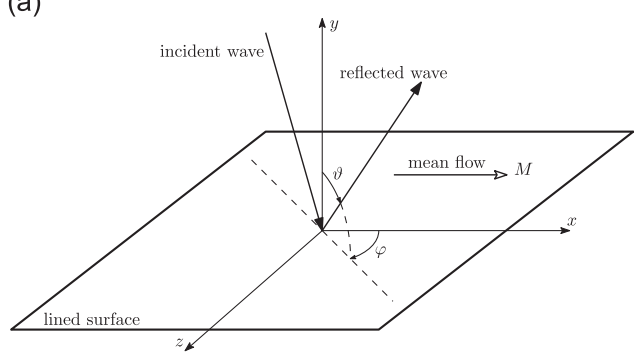

(b)

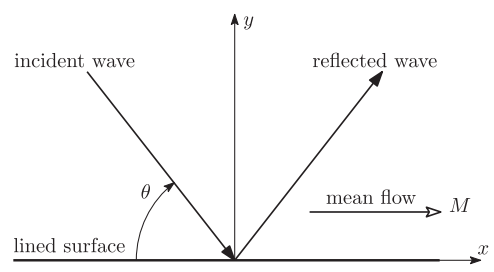

Fig. 1. Diagram of the incident and reflected wave in (a) three dimensions and (b) two dimensions. 


\subsection{Myers}

The Myers condition [2] assumes an infinitely thin boundary layer above the liner (continuity of pressure and normal displacement is imposed across this vortex sheet). The boundary condition reads

$$
-v=\frac{\mathrm{d}_{0}}{\mathrm{~d} t} \frac{p}{\mathrm{i} \omega Z}
$$

The corresponding reflection coefficient is given by (2) with $T_{0}=T_{1}=0$.

\subsection{Brambley}

The boundary condition proposed by Brambley [14] includes a boundary layer $0 \leq y \leq \delta$ above the impedance surface (the flow remains uniform above the boundary layer). The boundary layer thickness $\delta$ is assumed to be small but finite. Using matched asymptotic expansions and retaining leading-order terms in $\delta$ yields the following boundary condition (see Eq. (9) in Ref. [14]):

$$
-v\left[Z-\frac{\mathrm{i}}{\omega}\left(\omega-M k_{x}\right)^{2} \delta I_{0}\right]=\left[\frac{\omega-M k_{x}}{\omega}-Z \frac{\delta I_{1} k^{2} \sin ^{2} \vartheta}{\mathrm{i}\left(\omega-M k_{x}\right)}\right] p,
$$

where $k_{x}=k \sin \vartheta \cos \varphi$ is the streamwise wavenumber. It represents a boundary condition that is applied at $y=0$ to account for the effects of the boundary layer that are not modeled explicitly by (1). The coefficients $\delta I_{0}$ and $\delta I_{1}$ are given by

$$
\delta I_{0}=\int_{0}^{\delta} 1-\frac{\left[\omega-u_{0}(y) k_{x}\right]^{2} \rho_{0}(y)}{\left(\omega-M k_{x}\right)^{2}} \mathrm{~d} y, \quad \delta I_{1}=\int_{0}^{\delta} 1-\frac{\left(\omega-M k_{x}\right)^{2}}{\left[\omega-u_{0}(y) k_{x}\right]^{2} \rho_{0}(y)} \mathrm{d} y,
$$

where $u_{0}(y)$ and $\rho_{0}(y)$ denote the mean velocity and density profiles inside the boundary layer. The corresponding reflection coefficient is also of the form (2) with

$$
T_{0}=-k \delta I_{0} \cos \vartheta, \quad T_{1}=-k \delta I_{1} D \sin ^{2} \vartheta .
$$

As a special case, we consider a boundary layer with a uniform mean density and a mean velocity profile which is linear $u_{0}(y)=M y / \delta$ for $0 \leq y \leq \delta$ and then constant $u_{0}(y)=M$ for $y \geq \delta$. In this case we have

$$
T_{0}=\frac{1}{3} k \delta(D-1)(D+2) \cos \vartheta, \quad T_{1}=-k \delta(D-1) \sin ^{2} \vartheta .
$$

\subsection{Rienstra-Darau}

The boundary condition proposed by Rienstra and Darau [12,13] assumes a boundary layer with a small thickness $\delta$, a linear velocity profile and a uniform mean density. This two-dimensional boundary condition was derived in the incompressible limit and was devised to provide a good approximation of the hydrodynamic oscillations of the boundary layer (see also [15]). It reads

$$
\left(-\mathrm{i} \omega v+\delta \frac{\partial^{2} p}{\partial x^{2}}-\mathrm{i} \omega \sigma \delta \frac{\partial v}{\partial y}\right) Z=\frac{\mathrm{d}_{0} p}{\mathrm{~d} t}+\mathrm{i} \omega \delta\left[\mathrm{i} \omega(1-\sigma) v+(1-2 \sigma) M \frac{\partial v}{\partial x}\right]+\left(\frac{1}{3}-\sigma\right) \delta M^{2} \frac{\partial^{2} v}{\partial x^{2}}
$$

This defines a family of boundary conditions characterized by the parameter $\sigma$. Originally, $\sigma$ was set to zero [12], but subsequently it was suggested to use $\sigma=1 / 3$ to remove the second-order derivative in $v$ [13]. The case $\sigma=1$ is also considered here since it is more consistent with the special cases discussed in Section 3. The corresponding reflection coefficient is of the form (3) with

$$
T_{0}=k \delta \sin \theta\left[D-\sigma+\frac{1}{3}(D-1)^{2}\right], \quad T_{1}=k \delta\left[1+(\sigma D-1) \sin ^{2} \theta\right] .
$$

The generalization of Eq. (7) to three dimensions follows that in [13], and the derivation is outlined in Appendix A. However, it was found that the last step of the derivation differs from [13], and the following version of the boundary condition is proposed here:

$$
\left(-\mathrm{i} \omega v-\delta \frac{\partial^{2} p}{\partial y^{2}}-\mathrm{i} \omega \sigma \delta \frac{\partial v}{\partial y}\right) Z=\frac{\mathrm{d}_{0} p}{\mathrm{~d} t}+\mathrm{i} \omega \delta\left[\mathrm{i} \omega(1-\sigma) v+(1-2 \sigma) M \frac{\partial v}{\partial x}\right]+\left(\frac{1}{3}-\sigma\right) \delta M^{2} \frac{\partial^{2} v}{\partial x^{2}}
$$

The fundamental difference with (7) is the substitution of the term $\delta \partial^{2} p / \partial x^{2}$ on the left-hand side of (7) by $-\delta \partial^{2} p / \partial y^{2}$. The two alternatives are equivalent only when one considers a two-dimensional problem in the incompressible limit (in which case we have $\left.\partial^{2} p / \partial x^{2}+\partial^{2} p / \partial y^{2}=0\right)$. This is not an issue as far as modeling the hydrodynamic oscillations of the boundary layer is concerned. However, differences should be expected if Eqs. (7) and (9) are used to describe absorption of sound waves which obviously involves compressibility. Indeed, the two boundary conditions yield different results when used 
with the convected wave equation, and it will be shown in Section 4 that Eq. (9) can lead to more consistent predictions of sound absorption compared to (7). With the three-dimensional impedance condition (9), the reflection coefficient $R$ is of the form (2) with

$$
T_{0}=k \delta \cos \vartheta\left[D-\sigma+\frac{1}{3}(D-1)^{2}\right], \quad T_{1}=k \delta(\sigma D-1) \cos ^{2} \vartheta
$$

\section{Special cases}

Several special cases are now considered to provide some insight into the impedance boundary conditions and to assess their consistency.

\subsection{No flow}

We should obviously expect the boundary conditions (4), (5), (7) and (9) to reduce to the standard impedance condition $p=-Z v$ in the absence of mean flow $(M=0$, which also implies $D=1)$. This is indeed the case with the Myers condition (4). With the boundary condition (5) the terms $\delta I_{0}$ and $\delta I_{1}$ vanish when $u_{0}=0$ and $\rho_{0}=1$, and one recovers the standard impedance condition.

With Eq. (7) the terms $T_{0}$ and $T_{1}$ defined by (8) remain non-zero in the no flow case for any $\sigma$. As a consequence the standard impedance condition is not directly recovered, unless $\delta$ is explicitly set to zero. This is rather surprising since the parameter $\delta$ is only relevant for the cases with flow, and one would expect the boundary condition to be independent of $\delta$ in the no flow case. This is also the case with the modified boundary condition (9), except that the no flow case can be recovered if we set $\sigma=1$ so as to get $T_{0}=T_{1}=0$ when $D=1$.

\subsection{Waves propagating perpendicular to the mean flow}

The propagation of sound waves through the boundary layer is governed by the Pridmore-Brown equation:

$$
\frac{\partial^{2} p}{\partial y^{2}}+\left(\frac{2 u_{0}^{\prime} k_{x}}{\omega-u_{0} k_{x}}-\frac{\rho_{0}^{\prime}}{\rho_{0}}\right) \frac{\partial p}{\partial y}+\left[\left(\frac{\omega-u_{0} k_{x}}{c_{0}}\right)^{2}-k^{2} \sin ^{2} \vartheta\right] p=0 .
$$

If we assume a uniform mean density profile $\left(\rho_{0}=1\right)$ and an incoming plane wave propagating perpendicular to the mean flow (that is $\varphi= \pm \pi / 2$ which implies $k_{x}=0$ and $D=1$ ), then all mean flow effects vanish and Eq. (10) reduces to the standard wave equation. This holds irrespective of the boundary layer thickness and profile. In these special cases the mean flow and the boundary layer have no effect on the solution and the corresponding boundary condition is the same as with no flow: $p=-Z v$.

From the generic expression (2) for the reflection coefficient one can derive

$$
-v=\frac{1-\mathrm{i} Z T_{1}}{Z \cos \vartheta+\mathrm{i} T_{0}} p \cos \vartheta
$$

when $D=1$. For the Myers condition (4) we have $T_{0}=T_{1}=0$ so the condition $p=-Z v$ is recovered. The boundary condition (5) proposed by Brambley does capture this special case for an arbitrary velocity profile $u_{0}(y)$ since $\delta I_{0}=\delta I_{1}=0$ when $k_{x}=0$ and $\rho_{0}$ is uniform. With Eq. (9) we get $T_{0}=0$ and $T_{1}=k \delta(\sigma-1)$ so again this impedance condition is only consistent when $\sigma=1$. The boundary condition (7) applies in two dimensions, in which case we are restricted to $\vartheta=0$ and $\theta=\pi / 2$. We have $T_{0}=k \delta(1-\sigma)$ and $T_{1}=k \delta \sigma$ so the standard impedance condition cannot be recovered, irrespective of the choice of $\sigma$.

\subsection{Hard wall}

Considering the case of a hard wall might not seem relevant to discuss impedance conditions but it does provide some insight into some of these conditions. For a hard wall $(Z \rightarrow \infty)$ the Myers condition imposes a zero normal velocity. The situation is different for the alternatives proposed by Brambley and Rienstra \& Darau. Taking $Z \rightarrow \infty$ in (2) we find

$$
R=\frac{D \cos \vartheta+\mathrm{i} T_{1}}{D \cos \vartheta-\mathrm{i} T_{1}} \text { and }-v=\frac{-\mathrm{i} T_{1}}{D} p .
$$

For instance for the boundary condition (5) we get $-v=\mathrm{i} k \delta I_{1} \sin ^{2} \vartheta$. This means that if $T_{1} \neq 0$ the incoming acoustic wave is able to induce oscillations of the boundary layer. This is not an issue with the boundary conditions (5), (7) or (9), but rather a consequence of the finite boundary layer thickness $\delta$ described by these models. A possible interpretation of the terms $T_{1}$ and $\delta I_{1}$ is therefore that they represent the effective impedance of the boundary layer in isolation, as seen by the incident sound field. In fact inspection of Eq. (11) suggests that the effect of the boundary layer over a hard wall is equivalent to using a purely reactive admittance $1 / Z=-\mathrm{i} T_{1}$ in the Myers condition (4). For typical frequencies of interest this admittance will be small compared to the admittance of the liner. But for high frequencies, the admittance of the liner 
Table 1

Test cases considered in the comparison of the impedance conditions.

\begin{tabular}{llllll}
\hline Case & Helmholtz number $\omega$ & BL thickness $\delta(\%)$ & Mach number $M$ & Impedance $Z$ & $\omega \delta$ \\
\hline A & 28 & 1.4 & 0.55 & $5-\mathrm{i}$ & 0.39 \\
B & 28 & 0.7 & 0.55 & $5-\mathrm{i}$ & 0.2 \\
C & 56 & 1.4 & 0.55 & $5-\mathrm{i}$ & 0.78 \\
D & 28 & 1.4 & 0.3 & $5-\mathrm{i}$ & 0.39 \\
E & 28 & 3 & 0.55 & $3-0.5 \mathrm{i}$ & 0.84 \\
\hline
\end{tabular}

decreases and the impedance of the boundary layer alone will become significant. Finally, note that we have $|R|=1$ and $\alpha=0$ so the effect of the boundary layer is not to absorb sound but only to change the phase of the reflected wave.

\section{Results}

\subsection{Two-dimensional analysis}

The impedance boundary conditions are now compared using a series of two-dimensional test cases $\left(\varphi=0^{\circ}\right.$ or $\left.90^{\circ}\right)$ with parameters representative of turbofan engines. These parameters are listed in Table 1. Case A corresponds to the inlet of a typical turbofan engine at the blade passing frequency (BPF) and with Mach number $M=0.55$ and impedance $Z=5-\mathrm{i}$ (the curvature of the duct is neglected). The boundary layer thickness $\delta$ is 1.4 percent of the fan radius which is similar to what would be observed close to the fan. Several variants of this situation are also defined by considering a thinner boundary layer found further upstream of the fan (case B), by doubling the frequency (case C), or by considering a lower Mach number (case D). Finally case E corresponds to a bypass duct where the boundary layer can be much thicker and the impedance is different. In all cases we assume a linear boundary layer profile.

Also shown in Table 1 is the Helmholtz number $\omega \delta$ based on the boundary layer thickness. All the impedance conditions presented above assume that $\omega \delta \ll 1$. Table 1 shows that in practice this is not always true (especially cases $\mathrm{C}$ and $\mathrm{E}$ where the frequency or the boundary layer thickness are large). So it is not guaranteed that the impedance conditions will yield consistent results, and the present comparison will help gauge how accurate and robust these conditions are when applied to cases of practical interest. To that end the exact solution with a boundary layer with finite thickness and linear velocity profile is also obtained by solving numerically the Pridmore-Brown equation (10).

Results are presented in Figs. 2 and 3 by plotting the magnitude of the reflection coefficient $R$ and the absorption coefficient $\alpha$ as functions of the angle of incidence $\theta$. For the conditions (7) and (9) we consider the choices of parameter $\sigma=1$ and $1 / 3$ (other values of $\sigma$ have also been tested but these do not change the overall conclusions). Also, the results presented in Figs. 2 and 3 involve only the magnitude of the reflection coefficient, but the error on the phase was also investigated and the same conclusions can be drawn.

As a consistency check, we note that when $\omega \delta$ is small (case B) all impedance conditions tend to collapse onto the exact results, especially for downstream waves. As expected the boundary conditions (4), (5) and (9) with $\sigma=1$ yield the same results as the exact solution for normal incidence $\left(\theta=90^{\circ}\right)$ for all cases. Eq. (7) with either $\sigma=1$ or $\sigma=1 / 3$ tends to introduce significant differences at $90^{\circ}$. Eq. (9) with $\sigma=1 / 3$ is also different from the exact solution at normal incidence, although the difference appears quite small. The main conclusions are as follows:

Firstly, we discuss the effect of a finite boundary layer thickness (for lower frequencies similar assessments have been reported by Eversman [8] and Nayfeh et al. [9]). We see that the Myers condition tends to deviate significantly from the exact solution (particularly for cases A, C and E, but less so for cases B and D). This is mainly the case at the peaks of absorption, where the Myers condition overestimates the reflection coefficient. A general trend is that the effect of the boundary layer is stronger for upstream waves $\left(90^{\circ}<\theta<180^{\circ}\right)$. Note also that for more complex sound fields composed of a variety of plane waves with different directions, amplitudes and phases, the precise amount of absorption will depend to a large extent on the details of the incident sound field.

Secondly, the impedance condition (5) proposed by Brambley represents an improvement over the Myers condition in terms of predicting sound absorption. This has been observed for a wide range of configurations, and especially between $0^{\circ}$ and $130^{\circ}$, or for low Mach numbers (case D), where there is in fact very little difference with the exact solution. For angles greater than $130^{\circ}$, the differences are more significant but Eq. (5) still improves on the Myers condition. Also it seems to provide consistent results even when $\omega \delta$ is not very small (at high frequencies or for thick boundary layers), suggesting that this approximation for small $\omega \delta$ is relatively robust. Typical situations where the impedance condition (5) could improve the accuracy of current predictions based on the Myers condition is that of case A (an inlet where sound is propagating mostly upstream) and case E (a bypass duct where sound is propagating mostly downstream and the boundary layer can be relatively thick).

Thirdly, the boundary condition (7) proposed by Rienstra and Darau provides results quite different from the other solutions. The reflection coefficient is strongly overestimated for the complete range of angles of incidence. The most notable feature is that for upstream waves in cases $\mathrm{C}$ and $\mathrm{E}$, the boundary generates rather than absorbs acoustic energy. This leads to differences with the exact solution of 15 or $20 \mathrm{~dB}$ for the reflection coefficient. The modified version of the 
(A)

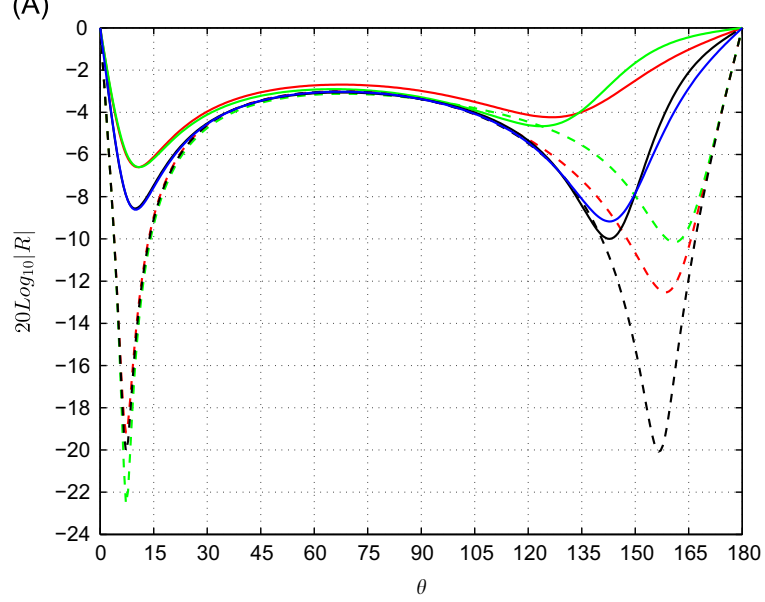

(C)

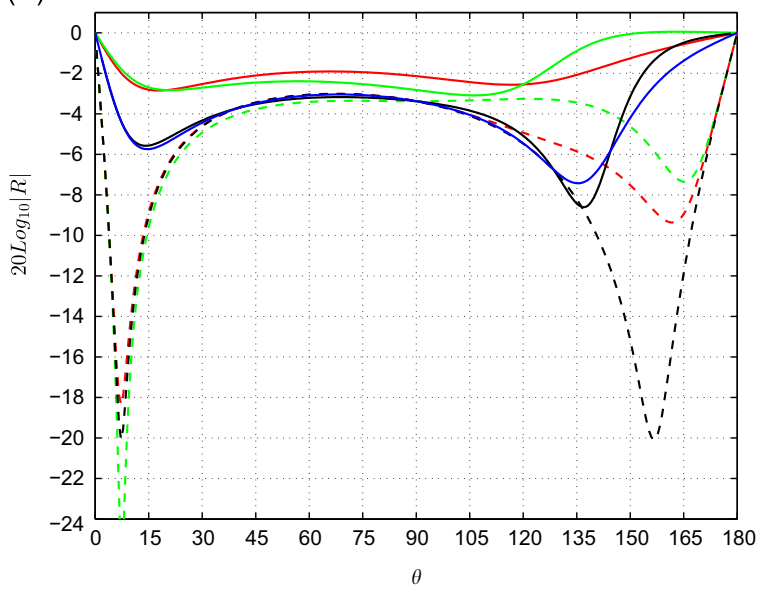

(B)

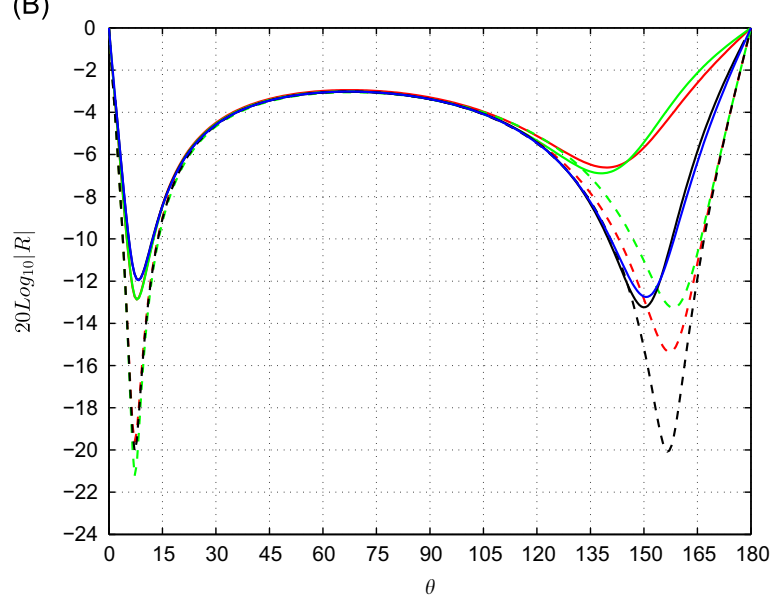

(D)

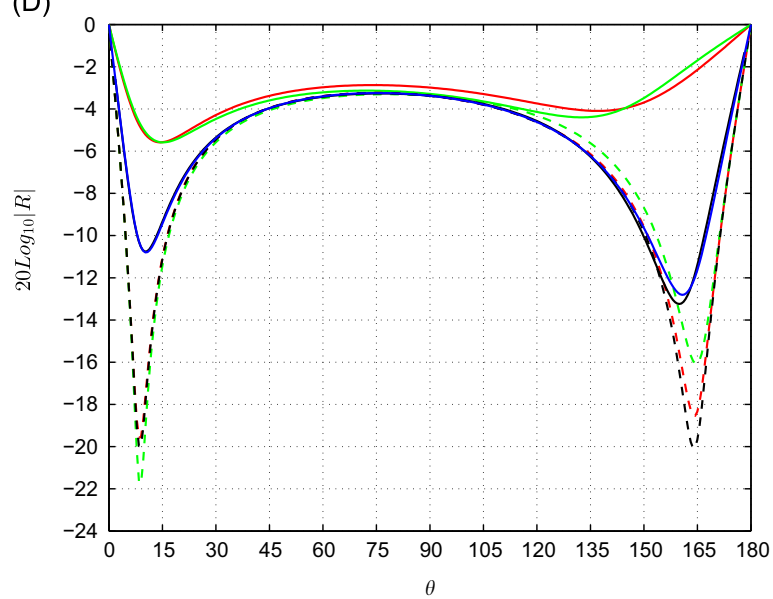

(E)

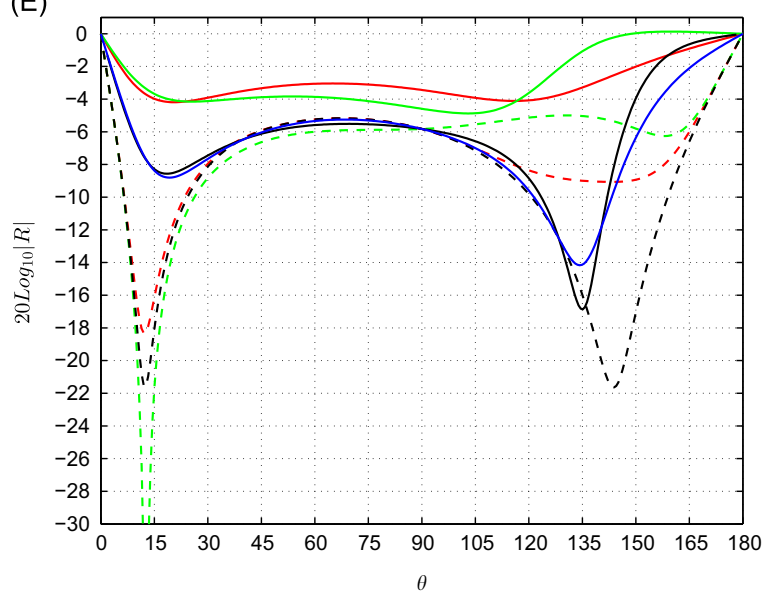

Fig. 2. Reflection coefficient $R$ (in $\mathrm{dB}$ ) for each case listed in Table 1. Solid black line: exact solution; dashed black line: Eq. (4); blue: Eq. (5); solid red line: Eq. (7) with $\sigma=1$; solid green line: Eq. (7) with $\sigma=1 / 3$; dashed red line: Eq. (9) with $\sigma=1$; and dashed green line: Eq. (9) with $\sigma=1 / 3$. (For interpretation of the references to color in this figure legend, the reader is referred to the web version of this article.) 
(A)

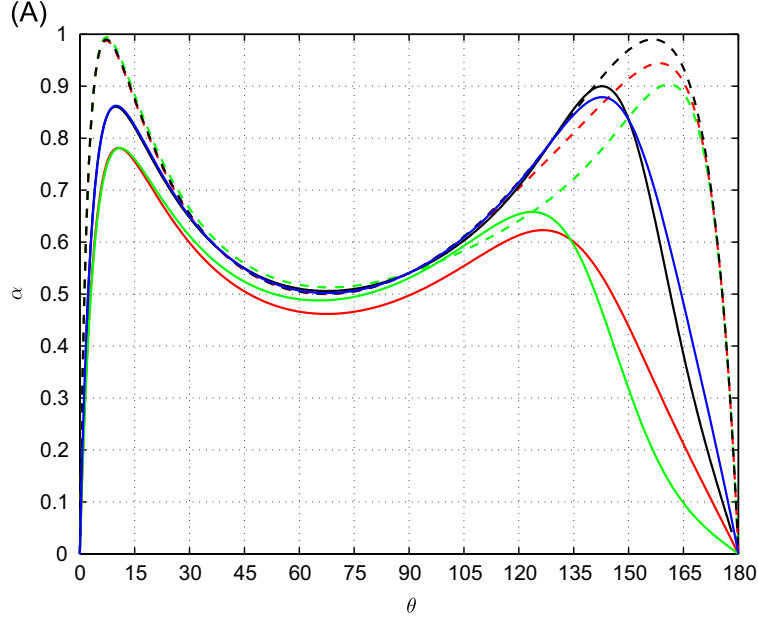

(C)

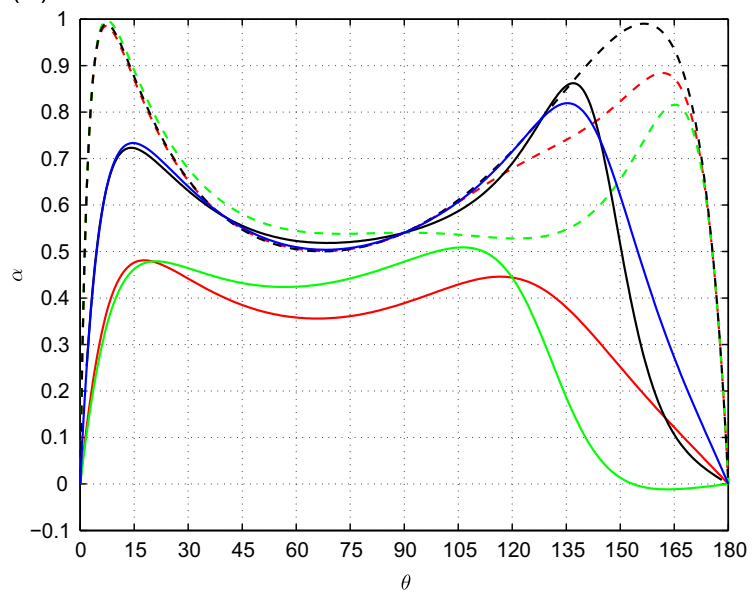

(B)

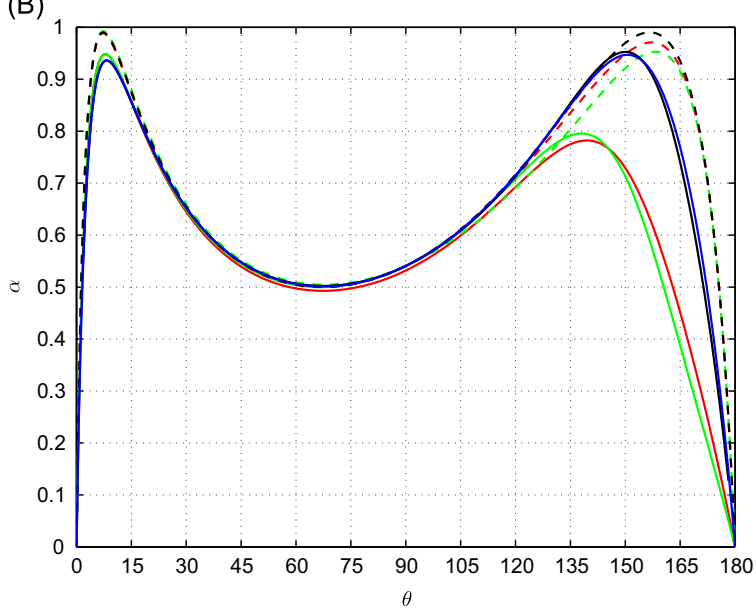

(D)

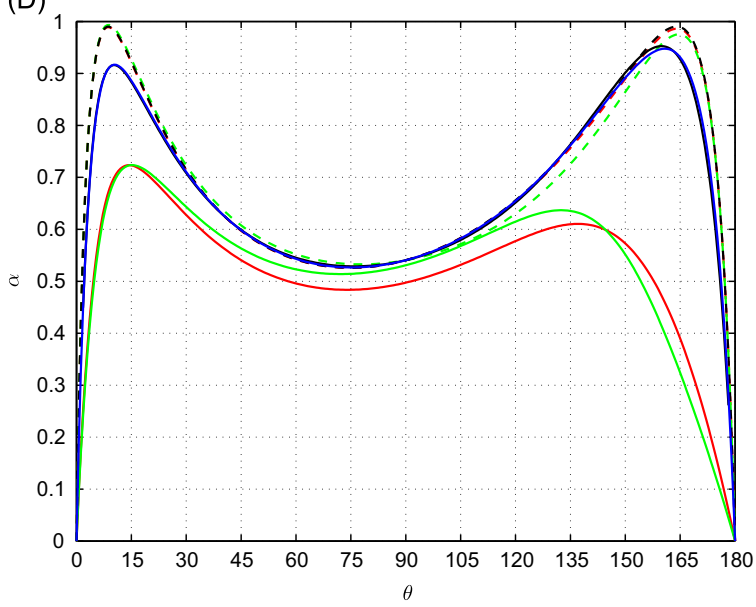

(E)

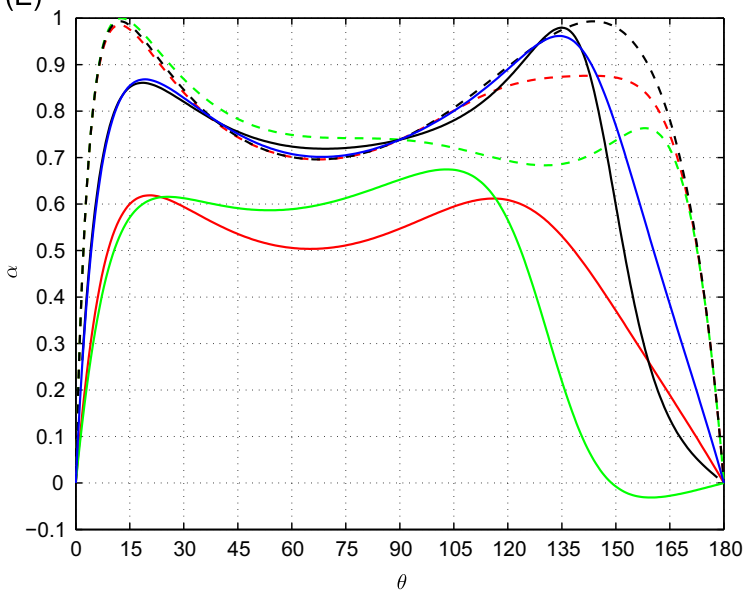

Fig. 3. Absorption coefficient $\alpha$ for each case listed in Table 1. Solid black line: exact solution; dashed black line: Eq. (4); blue: Eq. (5); solid red line: Eq. (7) with $\sigma=1$; solid green line: Eq. (7) with $\sigma=1 / 3$; dashed red line: Eq. (9) with $\sigma=1$; and dashed green line: Eq. (9) with $\sigma=1 / 3$. (For interpretation of the references to color in this figure legend, the reader is referred to the web version of this article.) 
(a)

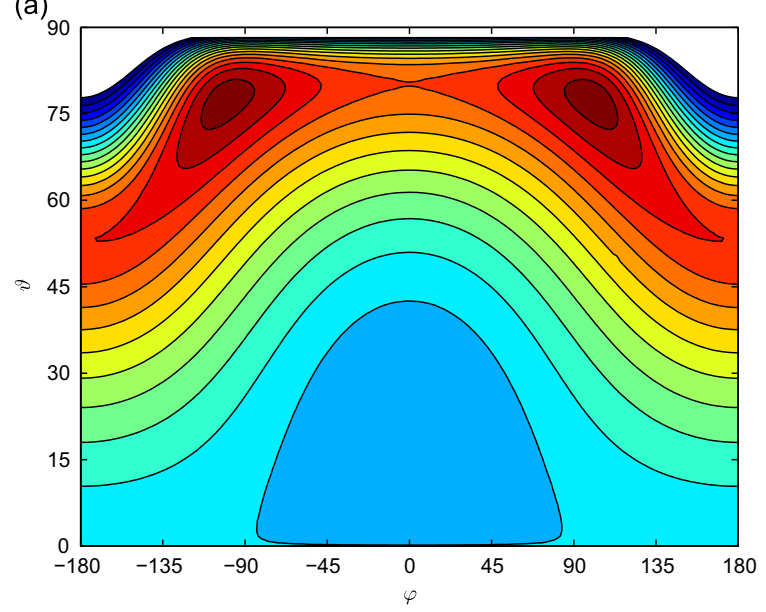

(c)

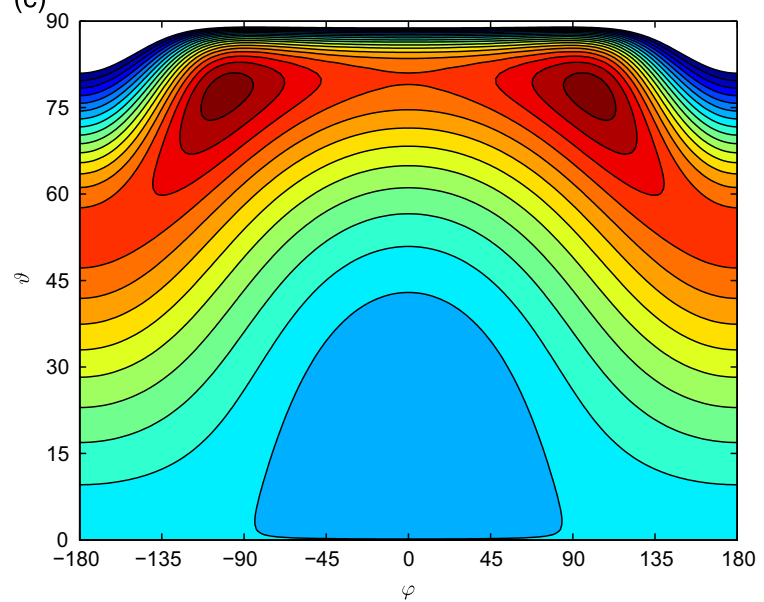

(b)

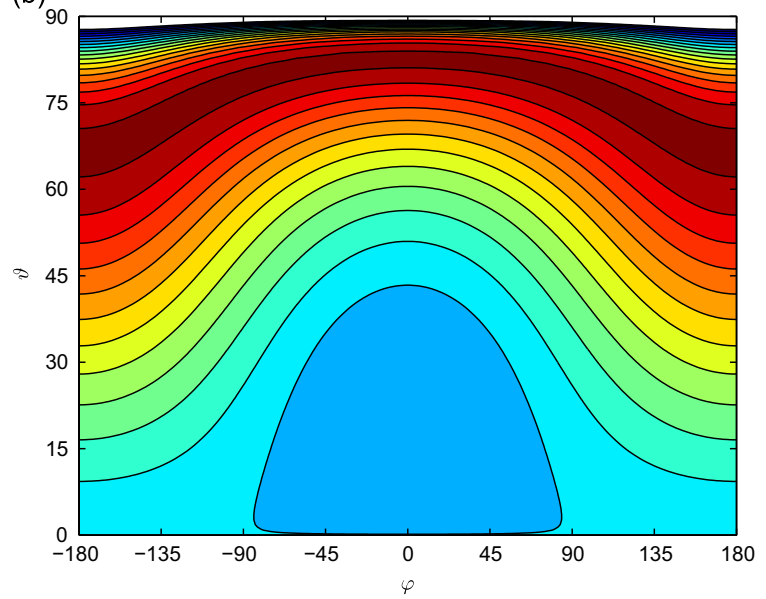

(d)

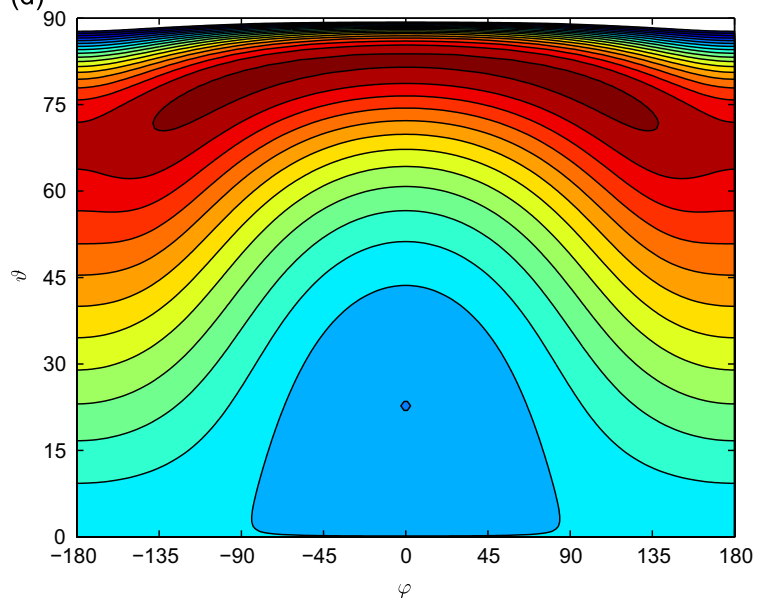

Fig. 4. Absorption coefficient $\alpha$ for case A as a function of $\varphi$ and $\vartheta$. Contour lines from 0.3 (blue) to 1.0 (red) spaced evenly every 0.04 : (a) exact solution; (b) the Myers condition (4); (c) Eq. (5); and (d) Eq. (9) with $\sigma=1$. (For interpretation of the references to color in this figure legend, the reader is referred to the web version of this article.)

Rienstra-Darau condition (9) does not suffer from this issue. But compared to the Myers condition it does not provide a significant improvement (in fact for downstream waves it tends to follow very closely the Myers results). This discrepancy can be explained by the fact that $\omega \delta$ is not necessarily small in the cases considered here, and the range of applicability of Eqs. (7) and (9) appears to be limited to very small values of $\omega \delta$. Also the effect of compressibility is neglected from the outset in the derivation of Eqs. (7) and (9). While this assumption is acceptable when modeling the hydrodynamic oscillations of the boundary layer (as shown in Ref. [13,15]), it is an issue if this boundary condition is used to model sound absorption by a lined surface.

\subsection{Three-dimensional effects}

To provide a more complete picture of the significance of the boundary layer thickness and of the accuracy of the impedance conditions, we now consider three-dimensional effects by varying both $\vartheta$ and $\varphi$. The absorption coefficient is shown in Fig. 4 for case A, for the exact solution and for the impedance conditions (4), (5) and (9) with $\sigma=1$.

The exact solution in Fig. 4 shows that the combined effects of the mean flow and the direction of the incident wave result in a complex pattern. The Myers condition is unable to capture these effects, and overall tends to overestimate the absorption. Of all the impedance conditions considered here only Eq. (5) seems able to follow the overall trends observed in the exact solution.

As explained in Section 3.2 the mean flow and the boundary layer have no effect when $\varphi= \pm 90^{\circ}$. This is indeed the case in Fig. 4 for the exact solution and the impedance conditions (4) and (5). Therefore, Fig. 4 also illustrates that the 

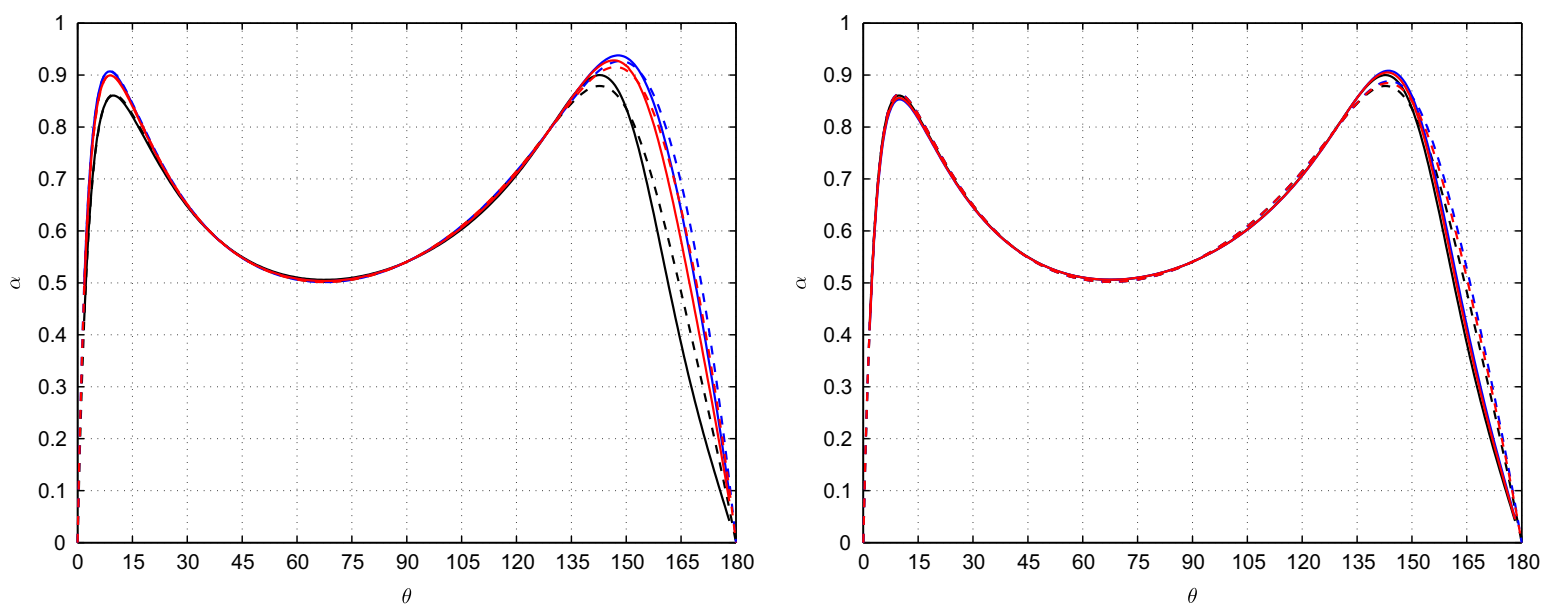

Fig. 5. Absorption coefficient $\alpha$ for case A with the linear profile (black), parabolic profile (red) and sinusoidal profile (blue). Solid lines: exact solutions; dashed lines: solutions obtained with Brambley's boundary condition. Left: the boundary layer thickness $\delta$ is the same for all profiles. Right: the displacement thickness $\delta_{1}$ is held constant for all profiles. (For interpretation of the references to color in this figure legend, the reader is referred to the web version of this article.)

influence of the boundary layer on sound absorption is strongest when the wave and the mean flow are parallel, and is non-existent when the two are perpendicular.

\subsection{Boundary layer profiles}

Finally we assess the significance of the boundary layer profile. Nayfeh et al. [9] conducted a comparison of the Myers condition against an exact solution for a finite boundary layer thickness. We present here a similar comparison but for cases more representative of modern turbofan engines - especially with higher frequencies - and we also consider the impedance condition derived by Brambley which is able to deal with arbitrary boundary layer profiles. So far we have used a linear profile, and we now introduce a parabolic profile

$$
u_{0}(y)=M-M(1-y / \delta)^{2} \quad \text { for } \quad y<\delta
$$

and a sinusoidal profile

$$
u_{0}(y)=M \sin (\pi y / 2 \delta) \text { for } y<\delta .
$$

For all three profiles we use a uniform mean density $\left(\rho_{0}=1\right)$.

For the impedance condition (5) we can write the term $\delta I_{0}$ as follows:

$$
\delta I_{0}=\frac{1}{\left(\omega-M k_{x}\right)^{2}}\left[\omega^{2} \delta_{0}-2 \omega k_{x} M \delta_{1}+M^{2} k_{x}^{2}\left(\delta_{1}+\delta_{2}\right)\right],
$$

where we have introduced the mass, displacement and momentum thickness of the boundary layer ${ }^{1}$ :

$$
\delta_{0}=\int_{0}^{\delta} 1-\rho_{0} \mathrm{~d} y, \quad \delta_{1}=\int_{0}^{\delta} 1-\frac{\rho_{0} u_{0}}{M} \mathrm{~d} y, \quad \delta_{2}=\int_{0}^{\delta} \frac{\rho_{0} u_{0}}{M}\left(1-\frac{u_{0}}{M}\right) \mathrm{d} y
$$

The term $\delta I_{1}$ has also to be calculated separately for each boundary layer profile.

Results are presented in Fig. 5 for the test case A. When the same boundary layer thickness $\delta$ is used for all profiles, it can be seen that some differences are visible, especially for upstream waves. Following the suggestion in [9], if we adjust $\delta$ in Eqs. (12) and (13) so that the displacement thickness $\delta_{1}$ is the same for all three profiles, then the results are almost identical and the actual boundary layer profile has in fact very little impact on sound absorption. This conclusion was also observed with the other test cases listed in Table 1. From a practical point of view, this indicates that the details of the boundary layer profile are not critical to obtain accurate predictions, and one can rely on macroscopic parameters such as the displacement thickness $\delta_{1}$. Results in Fig. 5 indicate also that the impedance condition (5) captures this feature quite well. As a consequence it is preferable to use the linear velocity profile since a simple expression for $\delta I_{1}=\delta M k_{x} / \omega$ is available in this case.

\footnotetext{
${ }^{1}$ We use here the usual definitions of the displacement and momentum thickness [16]. A different notation was used in [14].
} 


\section{Conclusions}

Two modified impedance conditions have been compared to the standard Myers condition and to an exact solution for the test case of a plane wave reflected off a flat lined surface, in two and three dimensions. The main observations are as follows:

- The effect of a finite thickness boundary layer can be significant, and the standard Myers condition can lead to significant errors when predicting sound absorption.

- The impedance condition proposed by Rienstra-Darau yields results that can be quite different from the exact solutions. This is due to the assumption of incompressibility that is used in the derivation of this condition. A variant of this impedance condition was proposed in three dimensions but it also suffers from the same issue.

- The impedance condition proposed by Brambley is able to improve on the predictions obtained with the Myers condition, even when the boundary layer is not very small compared to the acoustic wavelength.

- The details of the boundary layer profile have little effect on sound absorption, and it is sufficient to rely on parameters such as the displacement thickness to characterize the boundary layer.

Another modified Myers condition was proposed by Aurégan et al. [17] where an additional parameter is introduced to account for the effects of the viscous boundary layer. This impedance condition has not been considered here since the other impedance conditions and the exact solution do not include viscous effects. This could indeed represent an extension of the present comparison.

The results presented here are for a flat lined surface, but they can be carried across to a cylindrical duct since each duct mode has a ray direction that corresponds to specific values of $\varphi$ and $\vartheta$. As mentioned above the precise amount of absorption achieved by a given liner will depend on the modal content of the noise source.

\section{Appendix A. Derivation of Eq. (9)}

The same procedure as in Ref. [13] is followed here to obtain the three-dimensional version of Eq. (7), except for the last step which differs from Ref. [13]. We assume a uniform mean density and a sound field of the form $p=\hat{p}(y) \exp \left(i \omega t-\mathrm{i} k_{x} x-\mathrm{i} k_{z} z\right)$. The propagation of the sound field inside and outside the boundary layer is described by

$$
\hat{p}^{\prime \prime}+\frac{2 k_{x} u_{0}^{\prime}}{\omega-k_{x} u_{0}} \hat{p}^{\prime}+\left[\left(\frac{\omega-k_{x} u_{0}}{c_{0}}\right)^{2}-k_{x}^{2}-k_{z}^{2}\right] \hat{p}=0 .
$$

The derivation is based on the assumption that compressibility is negligible so the term $\left(\omega-u_{0} k_{x}\right) / c_{0}$ can be neglected.

Above the boundary layer $(y \geq \delta)$ the mean velocity $u_{0}$ is constant and Eq. (A.1) reduces to the Laplace equation. It follows that the solution for $y \geq \delta$ is $\hat{p}(y)=C_{1} \mathrm{e}^{-k_{y} y}$ with $k_{y}=\sqrt{k_{x}^{2}+k_{z}^{2}}$.

Inside the boundary layer $(0 \leq y \leq \delta)$ the mean velocity is varying linearly, $u_{0}(y)=M y / \delta$, and the following solution can be obtained:

$$
\hat{p}(y)=C_{2}\left[M k_{x}\left(k_{y} y-1\right)-\delta \omega k_{y}\right] \mathrm{e}^{k_{y} y}+C_{3}\left[M k_{x}\left(k_{y} y+1\right)-\delta \omega k_{y}\right] \mathrm{e}^{-k_{y} y} .
$$

The constants $C_{2}$ and $C_{3}$ are then eliminated by matching pressure and velocity at $y=\delta$. Finally we impose the standard impedance condition $p=-Z v$ at $y=0$ to obtain a dispersion relation:

$$
Z=\frac{\rho_{0}}{\mathrm{i} \delta} \frac{\delta \omega M k_{x} k_{y}\left[\left(2 k_{y} \delta+1\right) \mathrm{e}^{k_{y} \delta}-\mathrm{e}^{-k_{y} \delta}\right]-M^{2} k_{x}^{2}\left[\left(2 k_{y} \delta-1\right) \mathrm{e}^{k_{y} \delta}+\mathrm{e}^{-k_{y} \delta}\right]-2 \delta^{2} \omega^{2} k_{y}^{2} \mathrm{e}^{k_{y} \delta}}{k_{y}^{2}\left\{M k_{x}\left[\left(2 k_{y} \delta-1\right) \mathrm{e}^{k_{y} \delta}+\mathrm{e}^{-k_{y} \delta}\right]-2 \delta \omega k_{y} \mathrm{e}^{k_{y} \delta}\right\}} .
$$

To obtain an approximate boundary condition we can multiply both the numerator and denominator by $\mathrm{e}^{-k_{y} \delta \sigma}$ where $\sigma$ is a free parameter, and then expand each one independently in powers of $k_{y} \delta$ :

$$
Z \simeq \frac{\rho_{0}}{\mathrm{i}} \frac{\left(\omega-M k_{x}\right)^{2}+k_{y} \delta\left[\left(\frac{1}{3}-\sigma\right) M^{2} k_{x}^{2}+M k_{x} \omega(2 \sigma-1)+(1-\sigma) \omega^{2}\right]}{k_{y} \omega+k_{y}^{2} \delta\left[(1-\sigma) \omega-M k_{x}\right]} .
$$

Finally, the last step is to multiply both the numerator and denominator by $\hat{p}\left(k_{x}, k_{y}, k_{z}\right)$ and then convert the terms with $k_{x}$ and $k_{y}$ into spatial derivatives of $p(x, y, z)$. This leads to Eq. (9) where we have also used the linearized momentum equation to rewrite some derivatives of pressure in terms of velocity. This is where the difference with Eq. (7) originates from. In two dimensions, the dispersion relation for the solution is $k_{x}^{2}+k_{y}^{2}=0$ so we can substitute $\partial^{2} p / \partial x^{2}$ by $-\partial^{2} p / \partial y^{2}$. This is however not possible in three dimensions.

\section{References}

[1] K. Ingard, Influence of fluid motion past a plane boundary on sound reflection, absorption and transmission, Journal of the Acoustical Society of America 31 (7) (1959) 1035-1036. 
[2] M. Myers, On the acoustic boundary condition in the presence of flow, Journal of Sound and Vibration 71 (3) (1980) $429-434$.

[3] Y. Aurégan, M. Leroux, Experimental evidence of an instability over an impedance wall in a duct with flow, Journal of Sound and Vibration 317 (2008) 432-439.

[4] D. Marx, Y. Aurégan, H. Bailliet, J. Valière, PIV and LDV evidence of hydrodynamic instability over a liner in a duct with flow, Journal of Sound and Vibration 329 (2010) 3798-3812.

[5] S. Rienstra, A classification of duct modes based on surface waves, Wave Motion 37 (2003) 119-135.

[6] E. Brambley, N. Peake, Classification of aeroacoustically relevant surface modes in cylindrical lined ducts, Wave Motion 43 (2006) 301-310.

[7] E. Brambley, Fundamental problems with the model of uniform flow over acoustic linings, Journal of Sound and Vibration 322 (2009) $1026-1037$.

[8] W. Eversman, Effect of boundary layer on the transmission and attenuation of sound in an acoustically treated circular duct, Journal of the Acoustical Society of America 49 (5) (1971) 1372-1380.

[9] A. Nayfeh, J. Kaiser, B. Shaker, Effect of mean-velocity profile shapes on sound transmission through two-dimensional ducts, Journal of Sound and Vibration 34 (1974) 413-423.

[10] A. McAlpine, M. Fisher, B. Tester, "Buzz-saw" noise: a comparison of modal measurements with an improved prediction method, Journal of Sound and Vibration 306 (2007) 419-443.

[11] Y. Renou, Y. Aurégan, Failure of the Ingard-Myers boundary condition for a lined duct: an experimental investigation, Journal of the Acoustical Society of America 130 (1) (2012) 52-60.

[12] S. Rienstra, M. Darau, Mean flow boundary layer effects of hydrodynamic instability of impedance wall, IUTAM Symposium on Computational AeroAcoustics for Aircraft Noise Prediction, Southampton, UK, 2010.

[13] S. Rienstra, M. Darau, Boundary-layer thickness effects of the hydrodynamic instability along an impedance wall, Journal of Fluid Mechanics 671 (2011) 559-573.

[14] E. Brambley, Well-posed boundary condition for acoustic liners in straight ducts with flow, AIAA Journal 49 (6) (2011) $1272-1282$.

[15] D. Marx, A piecewise linear mean flow model for studying stability in a lined channel, Journal of Sound and Vibration 331 (2012) $3809-3823$.

[16] S.B. Pope, Turbulent Flows, Cambridge University Press, 2000.

[17] Y. Aurégan, R. Starobinski, V. Pagneux, Influence of grazing flow and dissipation effects on the acoustic boundary conditions at a lined wall, Journal of the Acoustical Society of America 109 (1) (2001) 59-64. 